\title{
Non-response to Communication Technology Outreach for Beta-agonist Overuse in a Pragmatic Randomized Trial of Patients with Asthma
}

\author{
Marsha A. Raebel, PharmD ${ }^{1,2}$, Susan M. Shetterly, MS ${ }^{7}$, Glenn K. Goodrich, $\mathrm{MS}^{7}$, \\ Courtney B. Anderson, $\mathrm{MPH}^{7}$, Jo Ann Shoup, $\mathrm{PhD}^{7}$, Nicole Wagner, $\mathrm{MPH}^{7}$, and Bruce G. Bender, \\ $P h D^{3}$
}

'Institute for Health Research, Kaiser Permanente Colorado, Denver, CO, USA; ${ }^{2}$ Skaggs School of Pharmacy and Pharmaceutical Sciences, University of Colorado, Aurora, CO, USA; ${ }^{3}$ Division of Pediatric Behavioral Health, National Jewish Health, Denver, CO, USA.

KEY WORDS: asthma; outreach; beta-agonist; response; pragmatic trial; communication technology; telephone; electronic.

J Gen Intern Med 33(6):809-11

DOI: $10.1007 /$ s11606-018-4395-9

(C) Society of General Internal Medicine 2018

\section{INTRODUCTION}

Evidence suggests that communication technology applications can improve treatment adherence. ${ }^{1}$ However, a recent Cochrane Review concluded insufficient evidence exists to determine the effects of automated communication on managing chronic conditions such as asthma. ${ }^{2}$ Because not all patients are receptive to communication technology interventions, defining factors associated with non-response to electronic outreach can inform tailoring future interventions to increase effectiveness. As part of a pragmatic trial targeting asthma patients with too frequent refills of inhaled betaagonists ("overfill"), ${ }^{3}$ the objective of this work was to describe and compare patients who did versus did not respond to a communication technology outreach. A higher asthma medication ratio (AMR), defined as the ratio of asthma controller medications (numerator, e.g., inhaled corticosteroids) to total controller medications plus inhaled beta-agonists (denominator), is associated with better asthma outcomes. ${ }^{4}$ We hypothesized that patients who did not respond to outreach would have a lower AMR than patients who did respond.

\section{METHODS}

This work was conducted at Kaiser Permanente Colorado (KPCO), an integrated healthcare system with approximately 600,000 members in the Denver-Boulder area. In KPCO usual care, when a patient refills a beta-agonist more frequently than every 60 days, an asthma care coordinator (ACC) is notified through the electronic health record (EHR) for patient followup. Because there are many reasons a patient could overfill (e.g., extra inhaler for gym), knowledge of current asthma symptoms is important, but unavailable to the ACC until after $\mathrm{s} /$ he contacts the patient. The electronic outreach was designed to determine whether the patient currently had symptoms to guide further contact.

The study included members aged $\geq 18$ diagnosed with persistent asthma and without chronic obstructive pulmonary disease. Patients were randomized 1:1 to receive a question about symptoms by text/call (call if phone was not textenabled) or email if they overfilled from 2/9/2017 through $11 / 8 / 2017$. Patients who responded they had symptoms were contacted by an ACC; patients without symptoms were not contacted. Patients who did not respond were contacted by an ACC if the ACC had not contacted them in the last 90 days and there was EHR evidence of a recent exacerbation (i.e., asthma-related corticosteroid burst, urgent care or emergency department visit, or hospitalization).

We compared responders and non-responders on characteristics, asthma exacerbations, and AMR the year prior to outreach. In univariable comparisons, Chi-square, Fisher's exact, or the Mann-Whitney test was used. We ran a multi-variable model and selected variables that individually explained $\geq 5 \%$ of the full model log-likelihood ${ }^{5}$ for presentation in a smaller, adjusted model estimating Relative Risks (RR) and 95\% confidence intervals $(95 \% \mathrm{CI})$ of non-response. Statistical analyses employed SAS version 9.4 (SAS Institute Inc., Cary, NC).

The KPCO Institutional Review Board approved this study. The requirement for informed consent was waived.

\section{RESULTS}

Altogether, 420 of 4953 (8.5\%) patients randomized to text $(N=284) /$ call $(N=136)$ and 398 of $5046(7.9 \%)$ patients randomized to email overfilled a beta-agonist and received outreach. Non-responders had a lower mean AMR $(P=0.019)$ (Table 1). Other characteristics associated with non-response included email outreach $(P<0.001)$, less than high school education $(P=0.021)$, lower family income $(P<0.001)$, and missed appointments $(P=0.008)$. 
Table 1 Characteristics of patients with asthma who did and did not respond to automated electronic beta-agonist overfill outreach

\begin{tabular}{|c|c|c|c|}
\hline Characteristic* & $\begin{array}{l}\text { Responded } \\
N=407\end{array}$ & $\begin{array}{l}\text { Did not } \\
\text { respond } \\
N=411\end{array}$ & $\begin{array}{l}P \\
\text { value } \dagger\end{array}$ \\
\hline $\begin{array}{l}\text { Asthma medication ratio } \\
(\mathrm{AMR}), \text { mean (SD) }\end{array}$ & $0.46(0.28)$ & $0.42(0.27)$ & 0.02 \\
\hline \multicolumn{3}{|c|}{ Beta-agonist overfill outreach type, \% } & $<0.001$ \\
\hline Email & 40.0 & 57.2 & \\
\hline $\begin{array}{l}\text { Text/call (if phone was } \\
\text { not text-enabled) }\end{array}$ & 60.0 & 42.8 & \\
\hline Age in years, mean $(\mathrm{SD})$ & $49.2(15.2)$ & $48.0(16.0)$ & 0.33 \\
\hline Female, $N(\%)$ & $233(57.2)$ & $249(60.6)$ & 0.36 \\
\hline Race, \% & & & 0.36 \\
\hline Asian & 2.9 & 2.2 & \\
\hline African American & 5.4 & 7.3 & \\
\hline American Indian/Alaskan & 0.7 & 0.7 & \\
\hline \multicolumn{4}{|l|}{ Native } \\
\hline White & 73.0 & 66.7 & \\
\hline Other & 5.7 & 7.3 & \\
\hline Unknown & 12.3 & 15.8 & \\
\hline Hispanic ethnicity, $\%$ yes & 13.5 & 18.5 & 0.05 \\
\hline \multicolumn{3}{|c|}{ Body mass index (BMI) in $\mathrm{kg} / \mathrm{m}^{2}, \%$} & 0.47 \\
\hline$<18.5$ & 1.0 & 0.7 & \\
\hline $18.5-24.9$ & 17.9 & 20.4 & \\
\hline $25-29.9$ & 34.9 & 29.2 & \\
\hline $30-34.5$ & 22.1 & 23.6 & \\
\hline $35-39.5$ & 11.3 & 14.1 & \\
\hline$\geq 40$ & 12.8 & 11.7 & \\
\hline \multicolumn{3}{|l|}{ Tobacco use, $\%$} & 0.71 \\
\hline Current smoker & 9.1 & 10.7 & \\
\hline Former smoker & 32.9 & 31.4 & \\
\hline Never smoker & 57.5 & 56.9 & \\
\hline $\begin{array}{l}\text { Elixhauser comorbidity } \\
\text { score }\left({ }^{6}\right) \text {, mean (SD) }\end{array}$ & $5.4(9.3)$ & $6.3(9.8)$ & 0.17 \\
\hline $\begin{array}{l}\text { Less than high school } \\
\text { education, } \% \text { in census } \\
\text { block }\end{array}$ & 9.4 & 10.7 & 0.02 \\
\hline $\begin{array}{l}\text { Family income, median } \$ \\
\text { (5th, 95th \%ile) }\end{array}$ & $\begin{array}{l}85,421 \\
(42,083 \\
135,054)\end{array}$ & $\begin{array}{l}78,554 \\
(39,099 \\
128,494)\end{array}$ & $<0.001$ \\
\hline $\begin{array}{l}\text { Total number of } \\
\text { ambulatory visits per } \\
\text { patient, mean (SD) }\end{array}$ & $5.9(7.8)$ & $5.4(6.3)$ & 0.97 \\
\hline \multicolumn{3}{|c|}{ Ambulatory appointments missed, $\%$ of patients } & 0.01 \\
\hline None & 69.5 & 61.3 & \\
\hline 1 & 18.4 & 19.0 & \\
\hline$\geq 2$ & 12.0 & 19.7 & \\
\hline $\begin{array}{l}\text { Total number of asthma- } \\
\text { related after-hour visits, } \\
\text { emergency department } \\
\text { visits, and/or hospitaliza- } \\
\text { tions per patient, mean } \\
\text { (SD) }\end{array}$ & $0.1(0.3)$ & $0.1(0.4)$ & 0.66 \\
\hline $\begin{array}{l}\text { Beta-agonist canisters } \\
\text { dispensed, mean (SD) }\end{array}$ & $5.1(4.0)$ & $5.5(4.8)$ & 0.12 \\
\hline $\begin{array}{l}\text { Short-acting corticosteroid } \\
\text { inhalers dispensed, mean } \\
\text { (SD) }\end{array}$ & $6.2(5.7)$ & $5.5(5.3)$ & 0.15 \\
\hline $\begin{array}{l}\text { Oral/injectable } \\
\text { corticosteroid bursts, } \% \\
\text { with } \geq 1\end{array}$ & 17.4 & 20.9 & 0.22 \\
\hline
\end{tabular}

*Unless specified, characteristics were determined for the 12 months prior to outreach

†Chi-square or Fisher's exact test for dichotomous variables, MannWhitney test for continuous variables

In adjusted analysis, non-response was associated with email outreach (RR 1.41, 95\% CI 1.22, 1.61) and $\geq 2$ missed appointments (RR 1.28, 95\% CI 1.09, 1.51) (Table 2). RR indicated lower AMR and lower family income were associated with non-response, although both 95\% CI included 1.00 (Table 2).
Table 2 Relative Risks of not responding to an automated electronic beta-agonist overfill outreach among patients with asthma

\begin{tabular}{lll}
\hline \hline Characteristic & $\begin{array}{l}\text { Unadjusted } \\
\text { Relative Risk } \\
\mathbf{9 5 \% ~ C I ) ~}\end{array}$ & $\begin{array}{l}\text { Adjusted } \\
\text { Relative Risk } \\
\mathbf{9 5 \% ~ C I ) *}\end{array}$ \\
\hline $\begin{array}{l}\text { Higher asthma medication } \\
\text { ratio } \dagger\end{array}$ & $0.92(0.86,0.99)$ & $0.93(0.87,1.00)$ \\
$\begin{array}{l}\text { Beta-agonist outreach type } \\
\text { reference -text/phone) }\end{array}$ & $1.41(1.23,1.62)$ & $1.40(1.22,1.61)$ \\
$\begin{array}{l}\text { Hispanic ethnicity } \\
\text { (reference-no/unknown) }\end{array}$ & $1.19(1.01,1.40)$ & $1.15(0.97,1.36)$ \\
$\begin{array}{l}\text { Less than high school } \\
\text { education (reference-high } \\
\text { school or higher education) }\end{array}$ & $1.71(0.96,3.04)$ & $0.89(0.43,1.85)$ \\
$\begin{array}{l}\text { Higher median family } \\
\text { income }\end{array}$ & $0.89(0.83,0.96)$ & $0.91(0.83,1.00)$ \\
$\begin{array}{l}\text { Ambulatory appointments missed (reference-none) } \\
\text { 1 missed appointment }\end{array}$ & $1.08(0.90,1.30)$ & $1.08(0.91,1.29)$ \\
$\begin{array}{l}\text { 2 or more missed } \\
\text { appointments }\end{array}$ & $1.32(1.13,1.55)$ & $1.28(1.09,1.51)$ \\
\hline
\end{tabular}

*Includes 812 (of 818) patients because 6 patients had missing data for $\geq 1$ characteristic(s)

†Relative risk estimated for increment of $\sim 1$ standard deviation (0.3 for AMR; $\$ 30,000$ for income)

\section{DISCUSSION}

In this evaluation of non-response to communication technology outreach among adults with asthma, non-response was higher among patients who had lower AMR. In other words, patients who did not respond to outreach used relatively less asthma controller medications or relatively more asthma reliever medications than patients who responded. An implication of this finding is that nonresponders have higher likelihood of worse asthma outcomes. ${ }^{4}$ Non-response was also higher among patients who received email outreach compared to text/call, missed $\geq 2$ ambulatory appointments a year, had less than high school education, or had lower income. When these characteristics were all considered, the AMR findings were not conclusive; further work is needed to define relationships between adherence and response to communication technology outreach. This profile of non-responders suggests that further tailoring of communication technology outreach is necessary to engage some patients, for instance patients who miss ambulatory appointments.

Corresponding Author: Marsha A. Raebel, PharmD; Institute for Health Research Kaiser Permanente Colorado, Denver, CO, USA (e-mail: Marsha.A.Raebel@kp.org).

Funders This work was funded by grant 1RO1HL126125 from the National Heart, Lung, and Blood Institute (NHLBI).

\section{Compliance with Ethical Standards:}

Prior Presentations: None.

Conflict of Interest: The authors declare that they do not have a conflict of interest. 


\section{REFERENCES}

1. Bender BG. Technology interventions for nonadherence: new approaches to an old problem. J Allergy Clin Immunol Pract. 2017 . https://doi.org/ 10.1016/j.jaip.2017.10.029.

2. Posadzki P, Mastellos N, Ryan R, Gunn LH, Felix LM, Pappas Y, et al. Automated telephone communication systems for preventive healthcare and management of long-term conditions. Cochrane Database Syst Rev. 2016;12:CD009921.

3. Bender B. The breathe well program for adults with asthma. ClinicalTrials gov Identifier: NCT02761837. Available at: https://clinicaltrials.gov/ct2/
show/NCT02761837. US National Library of Medicine; 2017. Accessed 15 Feb 2018

4. Schatz M, Zeiger RS, Vollmer WM, Mosen D, Mendoza G, Apter AJ, et al. The controller-to-total asthma medication ratio is associated with patient-centered as well as utilization outcomes. Chest. 2006;130(1):4350 .

5. Harrell FE. Regression modeling strategies. New York: Springer; 2001.

6. Beta Elixhauser Comorbidity Software for ICD-10-CM Healthcare Cost and Utilization Project (HCUP). Available at: www.hcup-us.ahrq.gov/ toolssoftware/comorbidityicd10/comorbidity_icd10.jsp. Rockville, MD Agency for Healthcare Research and Quality; 2017. Accessed 15 Feb 2018. 